\title{
Competition between pairing correlations and deformation from the odd-even mass staggering of francium and radium isotopes
}

\author{
S. Kreim,,${ }^{1,2, *}$ D. Beck, ${ }^{3}$ K. Blaum, ${ }^{2}$ Ch. Borgmann, ${ }^{2, \dagger}$ M. Breitenfeldt, ${ }^{4}$ T. E. Cocolios,${ }^{1, \dagger}$ A. Gottberg,,${ }^{1}$ F. Herfurth, ${ }^{3}$ \\ M. Kowalska, ${ }^{1}$ Yu. A. Litvinov, ${ }^{3}$ D. Lunney, ${ }^{5}$ V. Manea, ${ }^{5}$ T. M. Mendonca, ${ }^{1}$ S. Naimi, ${ }^{6}$ D. Neidherr, ${ }^{3}$ M. Rosenbusch, \\ L. Schweikhard, ${ }^{7}$ Th. Stora, ${ }^{1}$ F. Wienholtz, ${ }^{7}$ R. N. Wolf, ${ }^{7,8}$ and K. Zuber ${ }^{8}$ \\ ${ }^{1}$ CERN, 1211 Geneva, Switzerland \\ ${ }^{2}$ Max-Planck-Institut für Kernphysik, Saupfercheckweg 1, 69117 Heidelberg, Germany \\ ${ }^{3}$ GSI Helmholtzzentrum für Schwerionenforschung GmbH, 64291 Darmstadt, Germany \\ ${ }^{4}$ KU Leuven, 3001 Leuven, Belgium \\ ${ }^{5}$ CSNSM-IN2P3-CNRS, Université Paris-Sud, 91406 Orsay, France \\ ${ }^{6}$ RIKEN Nishina Center for Accelerator-Based Science, 2-1 Hirosawa, Wako-shi, Saitama 351-0198, Japan \\ ${ }^{7}$ Institut für Physik, Ernst-Moritz-Arndt-Universität, 17487 Greifswald, Germany \\ ${ }^{8}$ Technische Universität Dresden, 01069 Dresden, Germany
}

(Received 28 May 2014; published 4 August 2014)

\begin{abstract}
The masses of ${ }^{222,224,226-233} \mathrm{Fr}$ and ${ }^{233,234} \mathrm{Ra}$ have been determined with the Penning-trap mass spectrometer ISOLTRAP at the ISOLDE facility at CERN, including the previously unknown mass and half-life of ${ }^{233} \mathrm{Fr}$. We study the evolution of the odd-even staggering of binding energies along the francium and radium isotopic chains and of its lowest-order estimator, $\Delta^{3}(N)$. An enhancement of the staggering of $\Delta^{3}(N)$ is observed towards neutron number $N=146$, which points to contributions beyond pairing correlations. These contributions are investigated in the Hartree-Fock and Hartree-Fock-Bogoliubov approaches, emphasizing the connections to the single-particle level density and nuclear deformation.
\end{abstract}

DOI: 10.1103/PhysRevC.90.024301

PACS number(s): 21.10.-k, 21.60.-n, 29.30.Ep, 29.38.-c

\section{INTRODUCTION}

Binding energies along an isotopic or isotonic chain exhibit an odd-even staggering (OES) illustrating the stronger binding of an even-particle-number system due to the attractive pairing interaction with respect to its odd-particle-number neighbors. The OES effect can be quantitatively obtained through finitedifference formulas, the simplest being the single-particle separation energy

$$
S_{N}^{Z}=E(Z, N-1)-E(Z, N)
$$

the energy needed to remove the last valence neutron from the nucleus. Here, $E(Z, N)$ denotes the ground-state (negative binding) energy and $Z(N)$ the number of protons (neutrons). A second common quantity is the so-called empirical pairing gap, the difference of the actual binding energy of an oddparticle number system to the presumed one comparing its two even-particle-number neighbors (cf. Fig. 2 of [1]).This can be approximated by the three-point estimator (considering neutrons only)

$$
\Delta^{3}(N)=\frac{(-1)^{N}}{2}[E(N-1)-2 E(N)+E(N+1)] .
$$

\footnotetext{
*skreim@cern.ch

†Present address: Uppsala University, Department of Physics and Astronomy, Box 516, SE-751 05 Uppsala, Sweden.

${ }^{\ddagger}$ Present address: University of Manchester, Manchester M13 9PL, United Kingdom.

${ }^{\S}$ Present address: Max-Planck-Institut für Kernphysik, Saupfercheckweg 1, 69117 Heidelberg, Germany.
}

In near-spherical nuclei, the OES is manifested by larger charge radii for even- $N$ systems compared to the average of their odd- $N$ neighbors along an isotopic chain. Charge-radii OESs are also known to reveal dramatic changes in nuclear deformation; see, e.g., [2-4].

In theory, the OES is associated with the pairing gap as described by the Bardeen-Cooper-Schrieffer (BCS) theory [5]. The description of the properties of odd nuclides has received considerable interest during recent years in the framework of mean-field theory [1,6-12], where the OES of binding energies is the main constraint for the pairing interaction. As such, efforts have been devoted to understanding the different contributions to the OES and designing precise estimators for the pairing gap. However, the OES is difficult to describe in mean-field theory, as recently shown for ${ }^{132} \mathrm{Sn}$ [12]. With its two doubly magic nuclides, the $Z=50$ chain is important for constraining mean-field interactions. An alternative view from shell-model calculations is offered by [13]. In this mass region, the dependence of OES on isospin has also been investigated [14,15].

The neutron-rich francium and radium isotopes are intriguing cases for studying the OES of nuclear binding energies and the different contributions to estimators of the pairing gap. In this work, we report on the first determination of mass and half-life of the new isotope ${ }^{233} \mathrm{Fr}$ recently discovered at the fragment separator FRS at GSI [16]. Its mass is determined to an uncertainty of $10^{-7}$ using high-precision Penning-trap mass spectrometry, and the half-life to an uncertainty of $10^{-1}$ from decay spectroscopy. We also report first Penning-trap mass measurements of ${ }^{228,231,232} \mathrm{Fr}$ as well as ${ }_{233,234} \mathrm{Ra}$. 
Crossing a region of strong quadrupole and presumed octupole deformation [17], the neutron-rich francium and radium isotopic chains are suitable for understanding the impact of ground-state correlations resulting from different interactions on trends of differential quantities such as the two-neutron separation energy

$$
S_{2 N}^{Z}=E(Z, N-2)-E(Z, N)
$$

and higher order. Furthermore, the connection between the lower-order finite-difference formulas and the deformed single-particle picture [6] as well as the contribution of the time-odd part of the energy functional [9] can be tested.

Of the pairing-gap estimators, the lower-order finite-difference formulas are known to contain spurious mean-field contributions, which do not necessarily express the normal OES effect and cause an OES of the estimators themselves. Higher-order formulas, involving as many as five different nuclides, were proposed to give a proper estimation of the gap [1], while in parallel these spurious contributions have been investigated [10].

Apart from refining pairing correlations in the mean-field approach [1], the interest in the OES has been driven by the less-often-studied, time-odd part of the energy functional, which becomes active in odd nuclides. So-called dynamic core-polarization effects resulting from the breaking of timereversal symmetry in odd nuclei [1,9] offer the possibility of better constraining the time-odd part of the energy functional and must be considered when fitting the pairing interaction [7]. The OES of binding energies as a deformation effect has also been a subject of interest [6], since it has long been known for nucleonic systems [18] and has been observed in metal clusters [19] or ultrasmall superconducting grains [20,21]. A possible link to the underlying single-particle picture has been proposed in the Hartree-Fock limit (no pairing) [6] and its validity subsequently investigated in the HartreeFock-Bogoliubov picture (taking pairing correlations into account) $[1,10]$.

Complementary to literature results, studying these effects in the heavy-mass region of the nuclear chart allows understanding the scaling of their strength with the size of the many-body system. We will thus analyze the behavior of two-neutron separation energies $S_{2 N}$ and of the three-point estimator for the neutron pairing gap $\Delta^{3}(N)$ as a function of neutron number $N$ in the framework of the Hartree-Fock (HF) and Hartree-Fock-Bogoliubov (HFB) approaches. The changes in slope of $S_{2 N}$ and the staggering of $\Delta^{3}(N)$ will be regarded with respect to the underlying single-particle picture as well as the evolution of deformation upon adding neutrons to the nucleus. Within this analysis, the kink in two-neutron separation energies around $N=132$ can be explained in light of quadrupole and octupole correlations.

\section{EXPERIMENTAL SETUP AND RESULTS}

The francium and radium isotopes are measured with the Penning-trap mass spectrometer ISOLTRAP [22] coupled to the online isotope separator ISOLDE [23] at CERN. The radionuclides are produced by bombarding a depleted uraniumcarbide target with a $1.4-\mathrm{GeV}$ proton beam. Radium has four protons less than the uranium isotopes of the production target, hence its isotopes are amongst the heaviest nuclides obtainable at an ISOL facility. In the case of the francium measurement, the target is kept at a high temperature of around $2000^{\circ} \mathrm{C}$ and the cavity at a low temperature of $1600^{\circ} \mathrm{C}$ to suppress the radium contamination. The neutral atoms are surface ionized in a tantalum cavity and mass separated by the GPS magnetic

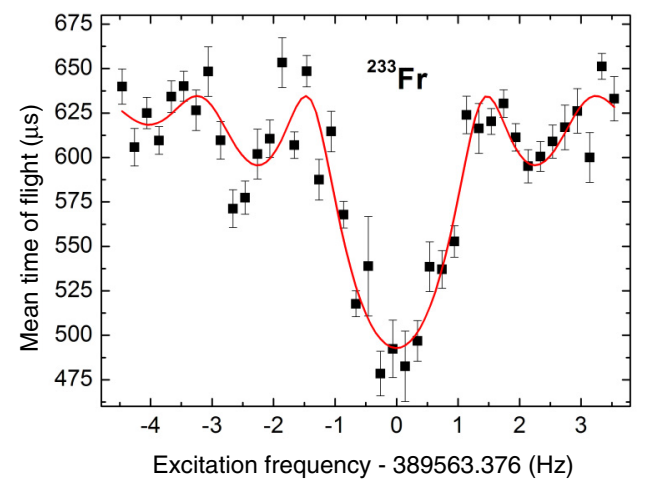

TOF detector

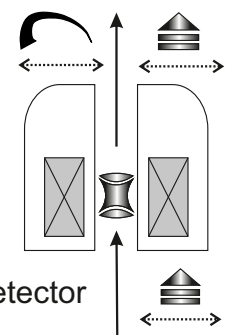

precision
Penning trap

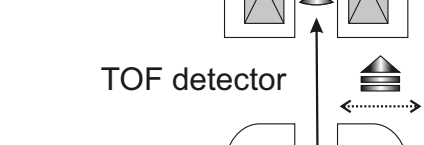

preparation

Penning trap

alkali

ion source
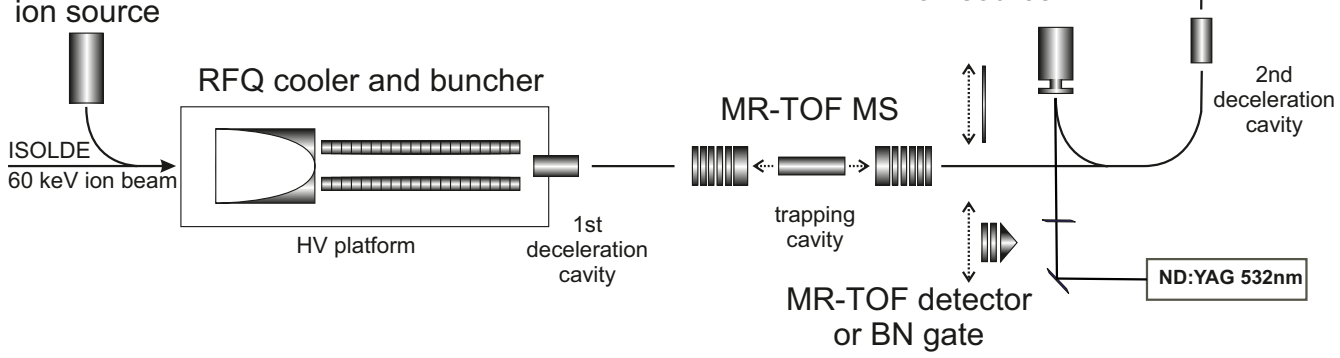

FIG. 1. (Color online) Schematic view of the ISOLTRAP setup. The inset shows a time-of-flight resonance of ${ }^{233} \mathrm{Fr}^{+}$. For details, see text. 
TABLE I. Number of TOF-ICR resonances taken, frequency ratio $r$, and mass excess $M E$ (in units of keV/c ${ }^{2}$ ) of the francium $(Z=87$ ) and radium $(Z=88)$ isotopes. The half-lives $\left(t_{1 / 2}\right)$ of the isotopes and the mass-excess values from the literature [35,36] are given $\left({ }^{\#}\right.$ marks extrapolated values, * has been determined within this work, and ${ }^{\dagger}$ marks the weighted mean). We compare to the atomic-mass evaluation (AME) from 2003 ( $\Delta_{\text {exp-lit }}$ ) because the main contribution to AME 2012 for the given nuclides comes from mass measurements of ESR [37], except for ${ }^{230} \operatorname{Fr}[38]$. The ESR data $\left(M E_{\mathrm{ESR}}\right)$ are given together with the difference to our data $\Delta_{\text {exp-ESR }}$.

\begin{tabular}{|c|c|c|c|c|c|c|c|c|c|}
\hline$A$ & $Z$ & $t_{1 / 2}$ & No. & $r$ & $M E_{\exp }$ & $M E_{\mathrm{AME} 03}$ & $\Delta_{\text {exp-lit }}$ & $M E_{\mathrm{ESR}}$ & $\Delta_{\text {exp-ESR }}$ \\
\hline 222 & 87 & $14.2(0.3) \mathrm{min}$ & 2 & $1.67049543(6)$ & $16378(7)$ & $16349(21)$ & $29(22)$ & & \\
\hline 224 & 87 & $3.33(0.1) \mathrm{min}$ & 5 & $1.68558717(9)$ & $21748(12)$ & $21660(50)$ & $88(51)$ & $21795(13)$ & $-47(18)$ \\
\hline 227 & 87 & $2.47(0.03) \mathrm{min}$ & 2 & $1.70822378(5)$ & $29682(7)$ & $29650(100)$ & $32(100)$ & $29686(13)$ & $-4(15)$ \\
\hline 228 & 87 & $38(1) \mathrm{s}$ & 3 & $1.71577790(6)$ & $33389(8)$ & $33280(200)^{\#}$ & $109(200)$ & $33367(15)$ & $22(17)$ \\
\hline 229 & 87 & $50.2(0.4) \mathrm{s}$ & 2 & $1.72332050(5)$ & & & & & \\
\hline 231 & 87 & $17.6(0.6) \mathrm{s}$ & 4 & $1.73842064(6)$ & $42080(8)$ & $42330(470)^{\#}$ & $-250(470)$ & $42064(25)$ & $16(26)$ \\
\hline 232 & 87 & $5.5(0.6) \mathrm{s}$ & 2 & $1.74597706(11)$ & $46073(14)$ & $46360(640)^{\#}$ & $-287(640)$ & & \\
\hline 233 & 87 & $0.9(0.1) \mathrm{s} *$ & 2 & $1.75352424(16)$ & $48920(20)$ & & & & \\
\hline 233 & 88 & $30(5) \mathrm{s}$ & 2 & $1.75348724(9)$ & $44339(12)$ & $44770(470)^{\#}$ & $-431(470)$ & $44322(16)$ & $17(20)$ \\
\hline 234 & 88 & $30(10) \mathrm{s}$ & 3 & $1.76103234(7)$ & $46931(8)$ & $47230(490)^{\#}$ & $-299(490)$ & $46893(31)$ & $38(32)$ \\
\hline
\end{tabular}

separator before being transported to the ISOLTRAP setup. Figure 1 shows the experimental setup, which consists of four traps: a linear segmented radio-frequency quadrupole ion beam cooler and buncher for beam preparation (RFQ cooler and buncher), a multireflection time-of-flight mass separator (MR-TOF MS), and two Penning traps (preparation and precision Penning trap) [24]. The 50-keV ion beam (up to $60 \mathrm{keV}$ is possible at ISOLDE) enters the ISOLTRAP setup by varying the opening time of the ISOLDE beam gate for mass measurements between $100 \mu$ s for the most abundant species on $A=222$ to $1 \mathrm{~s}$ for $A=234$, depending on the incoming ion current. The ion beam first enters the linear, gas-filled RFQ ion trap where buffer-gas cooling is applied for $20 \mathrm{~ms}$ [25]. After passing the first deceleration cavity, isobaric contaminants are separated due to mass-over-charge dependent flight times after repeated oscillations between the electrostatic mirrors of the MR-TOF MS [26]. A Bradbury-Nielsen gate (BN gate) [27] installed directly behind the MR-TOF MS reaches a suppression factor for contaminating ions of four orders of magnitude, thus considerably enhancing the performance of ISOLTRAP when dealing with large contamination ratios [28]. In this particular case, main contaminant and ion of interestin the case of radium francium and vice versa-exhibit a flight-time difference of a few hundred nanoseconds after 1000 revolutions at a total flight time of $t \approx 36 \mathrm{~ms}$, corresponding to a mass-resolving power of $R=m / \Delta m \approx 100000$. Yet, for the measurements presented here, the $\mathrm{BN}$ gate is not employed for purification purposes. In the helium buffer-gas filled preparation trap, the ion motion is cooled and the ion ensemble centered within a few hundred milliseconds by mass-selective resonant buffer-gas centering [29]. A francium-radium mixture is then sent to the second, precision Penning trap for the mass measurement. Here, the dipole-cleaning technique is used as sole purification method with an excitation time of $1 \mathrm{~s}$ to ensure a sufficiently small linewidth not to excite the ion of interest [30]. Contamination ratios are on the order of a few tens to one for francium as ion of interest. In the case of francium (radium) as ion of interest, the isobaric radium (francium) contamination is eliminated, while for ${ }^{234} \mathrm{Ra},{ }^{234} \mathrm{U}$ and its oxide (formed due to impurities in the buffer-gas environment of the preparation trap) are removed from the trap. The time-of-flight ion-cyclotron-resonance (TOF-ICR) technique is employed to determine the cyclotron frequency $v_{c}=q B /(2 \pi m)$ of a stored ion with mass $m$ and charge $q$ in a magnetic field $B$, from which the atomic mass $m_{a}$ can be extracted in conjunction with a reference mass measurement [31-33],

$$
m_{a}=\frac{v_{c}^{\mathrm{ref}, a}}{v_{c}}\left(m_{\mathrm{ref}, a}-m_{e}\right)+m_{e}=r\left(m_{\mathrm{ref}, a}-m_{e}\right)+m_{e} \text {. }
$$

Here, $m_{e}$ denotes the electron mass. For all measurements reported here, the reference ion is ${ }^{133} \mathrm{Cs}^{+}$, except for one of the measurements of ${ }^{229} \mathrm{Fr}$ where ${ }^{238} \mathrm{U}$ is used. Typical excitation times lie between $600 \mathrm{~ms}$ and $1200 \mathrm{~ms}$. Figure 1 shows two detectors for the TOF-ICR measurement: a multichannel plate (MCP) and a channeltron detector. For the measurements reported here, the MCP detector is used with which the detection rate for ${ }^{233} \mathrm{Fr}$ is $0.34(4)$ ions/s at a total cycle length of $3.83 \mathrm{~s}$. The overall efficiency from the merging switchyard of ISOLDE [23] to the detector is on the order of $10^{-3}$. The mass uncertainty is determined following [34].

Table I shows the frequency ratios and mass-excess values of the francium and radium isotopes measured within the scope of this work. It is also indicates how many measurements (TOF-ICR) were taken for each nuclide. The data are compared to literature values [36] and mass measurements from the storage ring ESR at GSI [37]. The literature values of the atomic-mass evaluation from 2003 are quoted because the main contributions to the ones from 2012 [39] for the given nuclides come from the ESR. ${ }^{1}$ In that paper, the authors introduce a novel data analysis with reduced systematic

${ }^{1}$ In the case of ${ }^{230} \mathrm{Fr}$ the main contribution comes from an ISOLTRAP value [38]. 
uncertainty. Comparing the two data sets $M E_{\text {exp }}$ and $M E_{\mathrm{ESR}}$ of Table I, four (one) out of the nine measurements from time-resolved Schottky-mass spectrometry fall out of the $1 \sigma$ $(2 \sigma)$ band ( $\sigma$ denotes the standard deviation).

The present measurements complement earlier ISOLTRAP measurements on ${ }^{220,223-229} \mathrm{Rn}, \quad{ }^{221,222,229,230} \mathrm{Fr}$, and ${ }^{226,229-232} \mathrm{Ra}[38,40-42]$. Please note that the results of Ref. [41] have already been included in the AME from 2003. In the case of ${ }^{228} \mathrm{Fr}$, one measurement was taken in an earlier run with the same target-ion-source combination. In the case of ${ }^{229} \mathrm{Fr}$, two TOF-ICR resonances were recorded taking ${ }^{133} \mathrm{Cs}$ as reference, the third with ${ }^{238} \mathrm{U}$ provided from the target itself. The mass-excess value is given as weighted mean. The discrepancy compared to the ISOLTRAP mass-excess value from Weber et al. of $M E_{A=229}=35816(37) \mathrm{keV}$ amounts to $4 \sigma$. The latter measurement can no longer be trusted due to close-lying contamination in the beam, as explained in [28].

In the case of ${ }^{233} \mathrm{Fr}$, mass and half-life of this isotope could be determined for the first time. Decays observed during mass measurements at ISOLTRAP lead to the preliminary conclusion of a half-life on the order of seconds, to explain the count-rate variation between the TOF-ICR measurements with different excitation times. To measure the ${ }^{233} \mathrm{Fr}$ half-life, the ISOLDE tape station is employed for beta-decay spectroscopy. The target and ion source unit is different from the one used for mass measurements reported here, i. e., conditions are set to suppress release and ionization of radium. The proton beam impinges on the target once in a $21 \mathrm{~s}$ period. After the proton pulse, the ion beam is implanted in a tape for $2 \mathrm{~s}$, after which the tape is moved to the detectors and the activity measured. Fifty separate beta-decay acquisitions are collected and summed up. Figure 2 shows the respective sum, after subtraction of background measured prior to implantation. To extract the half-life of the isotope, the experimental data is fitted using a double exponential decay function including the daughter feeding of ${ }^{233} \mathrm{Ra}$ with a fixed half-life of $30 \mathrm{~s}$ from ${ }^{233} \mathrm{Fr}$ where the half-life and the initial atomic ratio are left

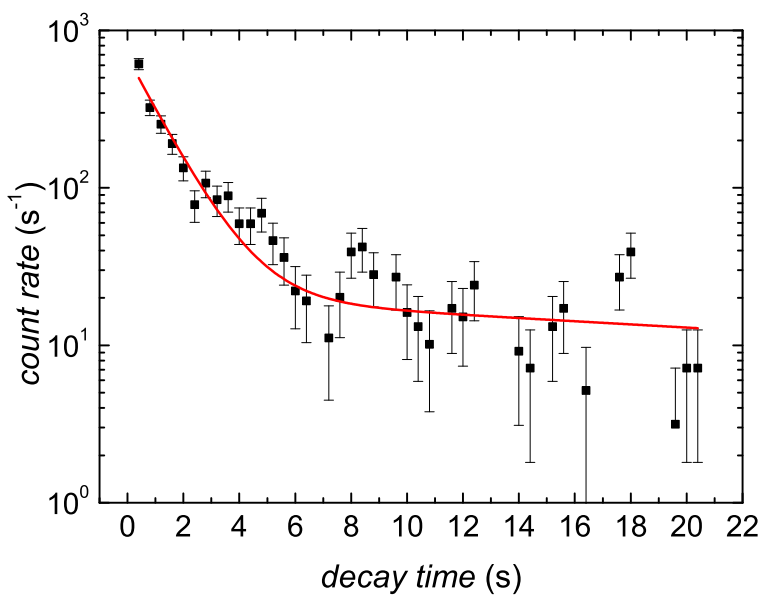

FIG. 2. (Color online) Beta-decay curve for the $A=233$ ISOLDE beam. Data from 50 separate acquisitions are added, and the average background is subtracted. A double exponential fit with daughter feeding is represented by the solid red curve. open in the fit. The fit yields a half-life of $(0.9 \pm 0.1) \mathrm{s}$ for ${ }^{233} \mathrm{Fr}$. Estimates for the half-life had been previously available only from theoretical predictions with values ranging from 0.3 to $14 \mathrm{~s}$ [43-45].

\section{DISCUSSION}

The francium and radium isotopes determined with the Penning-trap mass spectrometer ISOLTRAP lie in a region of the nuclear chart known for deformation, which becomes apparent from the peculiar behavior of two very common finite-difference formulas: the two-neutron separation energy $S_{2 N}$ and the empirical pairing gap $\Delta^{3}(N)$. The following discussion is thus divided in two parts. The first part treats the onset of deformation in the radium and francium isotopic chains and its impact on $S_{2 N}$ as well as the deformed singleparticle picture. The microscopic conditions for octupole collectivity will be discussed. In a second part, OES and contributions to the three-point estimator for the pairing gap $\Delta^{3}(N)$ are discussed.

The different existing theoretical approaches to the observed effects will be compared and their validity in the heavy-mass region discussed. To this end, HF and HFB calculations [46] have been performed using the HFODD code [47] and the SLy4 interaction [48,49]. The code solves the $\mathrm{HF}(\mathrm{B})$ equations in a harmonic-oscillator (HO) basis without imposing time-reversal symmetry. The starting conditions for the $\mathrm{HF}(\mathrm{B})$ quadrupole-deformed solutions are found by adding a constraint on the quadrupole moment to the energy functional for the first ten iterations $[47,50]$. A HFB calculation constraining the nuclei to spherical shape was also performed, serving as reference for the contribution of static deformation to the ground-state energy. A delta volume-pairing force was used [46], with the strength (equal for protons and neutrons) of $-200 \mathrm{MeV} \mathrm{fm}^{3}$, which gives a good description of the onset of quadrupole deformation in the neutron-rich region of the nuclear chart around $A=100$ [51].

\section{A. Deformation effects in the trends of two-neutron separation energies}

Figure 3 shows the two-neutron separation energy $S_{2 N}$ as a function of $N$ for the francium $(Z=87)$ and radium $(Z=$ 88 ) isotopic chains. The black open symbols mark the data from [39] including the ESR data listed separately in Table I. The ISOLTRAP data are shown in full symbols. The error bars lie in most cases within the symbol size. Exceptional occurrences in $S_{2 N}$ include the steep drop after the shell closure at $N=126$ as well as the change of slope beyond $N=132$ in the radium chain corresponding to a region of octupole collectivity [17]. Recently, this behavior has been investigated in the radon and radium isotopic chain producing evidence for static octupole correlations in radium [52]. Also, a bump emerges in the francium isotopic chain between $N=140$ and $N=146$ which will be treated in Sec. III B.

The lines in Fig. 3 represent different calculations of the mean-field approaches from this work and literature using either the SLy4 interaction or the Gogny D1S interaction. The odd and odd-odd nuclei presented in the figure are calculated 


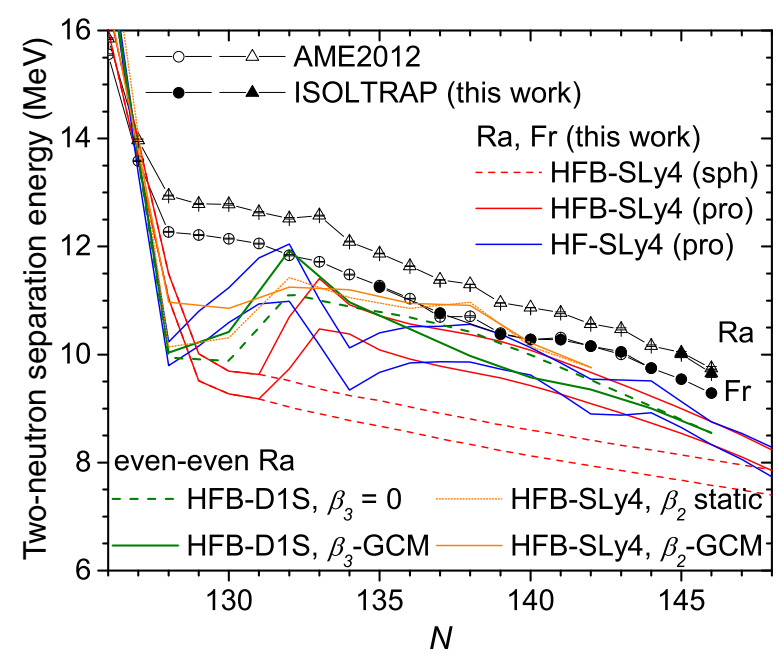

FIG. 3. (Color online) Two-neutron separation energy $S_{2 N}$ in the francium and radium isotopic chains. Experimental data from the AME [39] (open symbols) and ISOLTRAP (full symbols). The data are compared to spherical (statically deformed) HFB calculations depicted by dashed (solid) red lines and HF calculations with static deformation (solid blue lines). The thick green lines represent the HFB calculations with and without octupole correlations included (continuous and dashed, respectively) [55]. The thick orange lines represent BCS calculations [54] including only static deformation (dotted line) or also dynamic quadrupole correlations (continuous line).

at the HFB level as fully-paired states meaning that only pairs of interacting particles are considered, yet a state is created with an odd average particle number $[9,10]$. This is done without quasiparticle blocking, the method usually used to describe an odd-particle-number system: one of the particles has to be chosen not to be subject to pairing correlations; it is thus blocked from the pairing interaction. The spherical HFB solution is depicted by the dashed red line. The prolate solutions, obtained at the HF and HFB level, are plotted with continuous lines (blue and red, respectively). In general, two equilibrium prolate deformations are found, but of the two only the less deformed solution becomes more bound than the spherical one, the subsequent shape transition taking place around $N=132$. This is in agreement with the HFB Gogny calculation of [53]. As such, the continuous red line of Fig. 3 marks the lowest-energy (spherical or deformed) HFB configuration.

In the HFB picture, pairing correlations maintain the spherical shape of the nuclear ground states up to $N=131$, where the shape transition to prolate-deformed suddenly takes place. In the HF picture, deformation sets in more gradually from $N=128$ onwards. While the overall behavior of the two-neutron separation energies is similar in the HF and HFB calculations, the trend is much smoother in the HFB result.

The significant discrepancy on the absolute energy scale between the calculated and the experimental $S_{2 N}$ below $N=$ 132 is mainly due to missing dynamic quadrupole correlations, which requires going beyond the static mean-field approach by angular-momentum projection and configuration-mixing techniques [46]. To illustrate this, the results obtained for even-

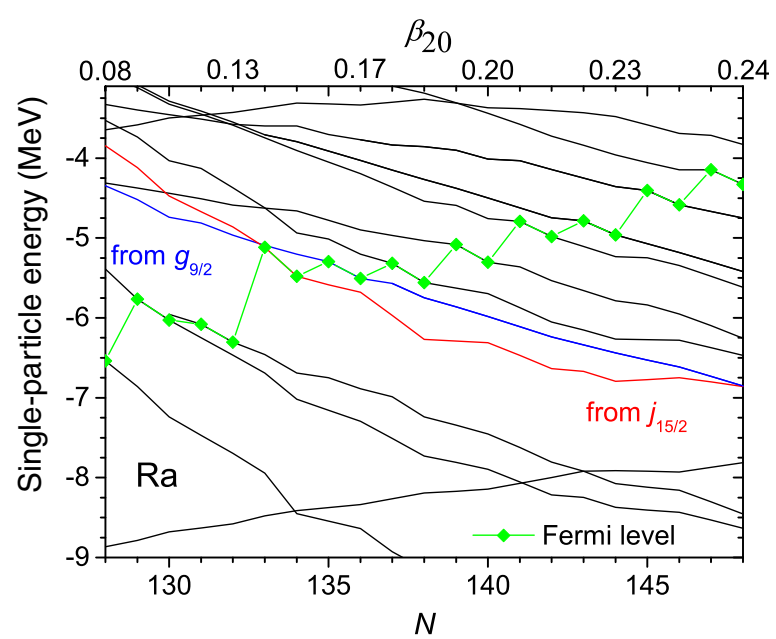

FIG. 4. (Color online) Neutron Nilsson levels of the radium isotopes between $N=128$ and $N=148$ from the HF calculation. For each $N$, the Nilsson levels of the corresponding isotope are presented. The Fermi level of each isotope is also shown.

even radium isotopes in the case of the SLy4 calculation of [54] are also shown, taking into account only static deformation as in the present work (dotted orange line) and including dynamic correlations by the generator-coordinate method (GCM, solid orange line). The smoothening of the transition at $N=132$ is obvious, although the calculated $S_{2 N}$ is still systematically lower than the experimental values. It can be concluded that the effect of quadrupole correlations along the represented isotopic chains is qualitatively already well described at the static mean-field level.

From the deformed HF calculations, the neutron Nilsson levels of the radium isotopes between $N=128$ and $N=148$ are calculated and shown in Fig. 4. The Nilsson orbits evolve

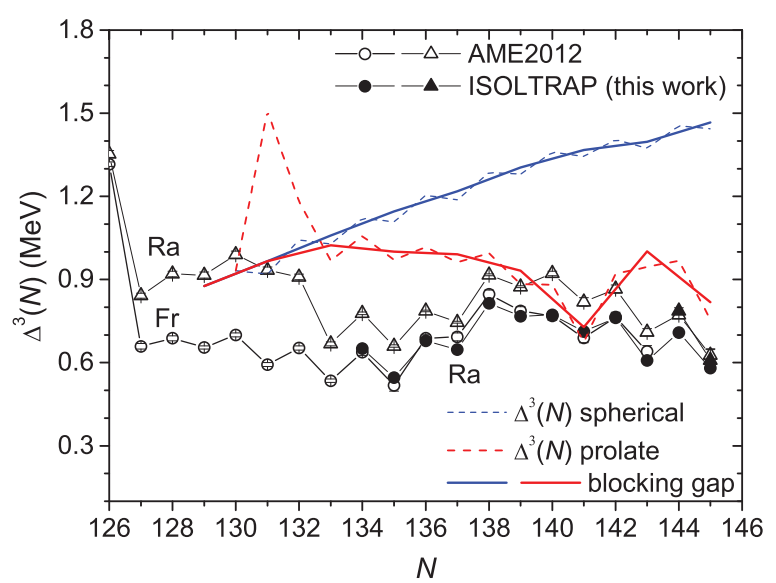

FIG. 5. (Color online) Three-point estimator of the pairing gap $\Delta^{3}(N)$ as a function of $N$ for the francium and radium isotopic chains. Data are compared to HFB calculations with quasiparticle blocking (for the radium isotopes) in the spherical and prolate-deformed approximations (blue dashed and red dashed lines, respectively). The new data show an enhancement of the staggering of $\Delta^{3}(N)$ towards neutron number $N=146$. 
with $N$ due to the change in quadrupole deformation and to the rearrangement of the mean field, forming gaps in the singleparticle energy spectrum as for example from $N=132$ to $N=133$. The large energy gap is accompanied by the crossing of two orbits originating from the $g_{9 / 2}$ and $j_{15 / 2}$ spherical shells. In the Nilsson picture, the lowering of the intruder level is the effect of quadrupole deformation (see upper abscissa). The two nearly-degenerate orbits have opposite parity and a difference in angular momentum of $\Delta j=3$ important for octupole coupling [17]. Sufficiently large energy gaps are reflected in changes of certain finite-difference formulas. Consider the energy gap between $N=144$ and $N=145$, which is about twice the size of the ones before. This is accompanied by a slope change in the $S_{2 N}$ values of the HF calculation (cf. Fig. 3).

A recent global HFB calculation using the Gogny interaction [55] includes static and dynamic octupole correlations by the GCM for even-even nuclei. Results for the radium chain are shown in Fig. 3 and exhibit an agreement similar to the HFB-SLy4 calculation. The inclusion of octupole correlations $\left(\beta_{3} \neq 0\right)$ enhances the peak at $N=132$. As a consequence, the full magnitude of the kink in $S_{2 N}$ at $N=132$, which is a maximum in the radium chain, can plausibly be explained as the combined effect of quadrupole and octupole correlations.

We note that the energy splitting of the Nilsson-orbit partners due to the breaking of time-reverseal symmetry in odd nuclides is predicted to increase monotonically from approximately $90 \mathrm{keV}$ at $N=131$ to roughly double the value at $N=147$. For simplicity, this splitting is not presented in Fig. 4, where only the Nilsson partner of higher energy is shown for each $N$. The effect is more than two times weaker than the one calculated for the cerium isotopes by Duguet et al. [9].

\section{B. The OES of nuclear masses and the three-point finite-difference formula}

The simplest phenomenological explanation of OES attributes it to the blocking effect of the odd nucleon, meaning that all particles but this one are subject to the pairing interaction. Bender et al. [1] denominate the size of this effect by the generic name of "blocking gap," and show that the dominant contribution originates from the blocking of pairing correlations. The blocking gap denotes the difference in binding energy of the odd-particle-number system to the value between its two even-particle-number neighbors (cf. Fig. 2 of [1]). Another contribution arises from polarization effects by the odd neutron, which causes a breaking of time-reversal and rotational symmetry $[1,7,9,56]$.

In the following, an expression for the three-point estimator of the pairing gap will be derived. For simplicity, we refer only to the OES as a function of neutron number $N$. Neglecting the dependence on proton number $Z$, the ground-state energy along an isotopic chain can be written as [57]

$$
E(N)=E_{0}(N)+\frac{(-1)^{N+1}}{2} D_{N},
$$

where $E_{0}$ is a term varying smoothly with neutron number, and $D_{N}$ is the neutron blocking gap. We note that for odd-odd nuclei an extra term describing the residual interaction between the odd proton and neutron would contribute, which is not considered.

Following the approach of $[1,10]$ the first term of Eq. (5) can be expanded in a Taylor series around a certain neutron number $N_{0}$ yielding for the ground-state energy

$$
\begin{aligned}
E(N)= & E_{0}\left(N_{0}\right)+\sum_{n=1}^{\infty} \frac{1}{n !}\left(\frac{\partial^{n} E_{0}}{\partial N^{n}}\right)_{N_{0}} \\
& \times\left(N-N_{0}\right)^{n}+\frac{(-1)^{N+1}+1}{2} D_{N} .
\end{aligned}
$$

From this, an approximate expression for the lowest-order finite-difference formula is given by the three-point estimator

$$
\Delta^{3}\left(N_{0}\right)=\frac{(-1)^{N_{0}}}{2}\left[E\left(N_{0}-1\right)-2 E\left(N_{0}\right)+E\left(N_{0}+1\right)\right] \text {. }
$$

By applying this filter to Eq. (5), one obtains an approximation for the blocking gap:

$$
\begin{aligned}
\Delta^{3}\left(N_{0}\right)= & D_{N}+\frac{(-1)^{N_{0}}}{2} \\
& \times\left[\left(\frac{\partial^{2} E_{0}}{\partial N^{2}}\right)_{N_{0}}+\frac{1}{12}\left(\frac{\partial^{4} E_{0}}{\partial N^{4}}\right)_{N_{0}}+\cdots\right],
\end{aligned}
$$

where $D_{N}$ is assumed to have a weak variation with neutron number.

The second term on the right-hand side of Eq. (8) represents the staggering of the three-point estimator around the value of the blocking gap. It is determined to leading order by the second derivative of the ground-state energy. The higher-order finite-difference formula $\Delta^{5}(N)$, eliminates the contribution of the second derivative and gives a better approximation of the blocking gap $D_{N}[1]$.

The experimental values of the empirical pairing gap $\Delta^{3}(N)$ using Eq. (7) are presented in Fig. 5. Measurement uncertainties are mostly smaller than the symbol size. Also shown are results for $\Delta^{3}(N)$ of the HFB calculations for the radium isotopes in the spherical (blue dashed line) and prolate-deformed (red dashed line) approximations with self-consistent quasiparticle blocking. The way blocking is implemented in the HFODD code is described in [50]. The parabolic behavior of $\Delta^{3}(N)$ in the spherical approximation, where only the pairing gap contributes, is suppressed by the effect of quadrupole deformation, which is essential to bring the theoretical prediction closer to the experimental values. Figure 5 also shows the blocking gap for the two calculations, i.e., the difference between the nonblocked and blocked odd- $N$ solution around which $\Delta^{3}(N)$ staggers. The prolate calculation does not correctly describe the experimental results around the onset of quadrupole deformation at $N=132$ as the suddenness of this effect is too large in the absence of dynamic correlations (as discussed in the previous subsection). However, the calculation comes close to experiment in the region beyond $N=136$.

Although the staggering of $\Delta^{3}(N)$ itself renders it less suitable for fitting the pairing interaction [1], the authors 


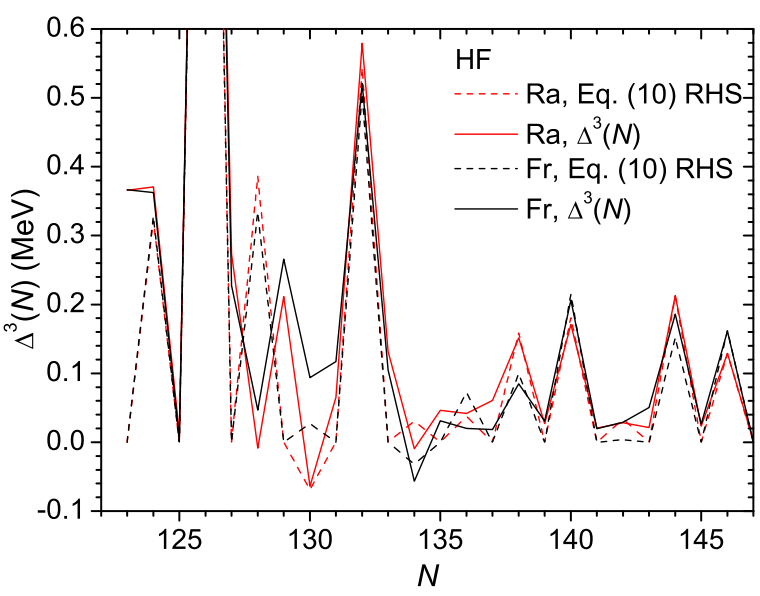

(a)

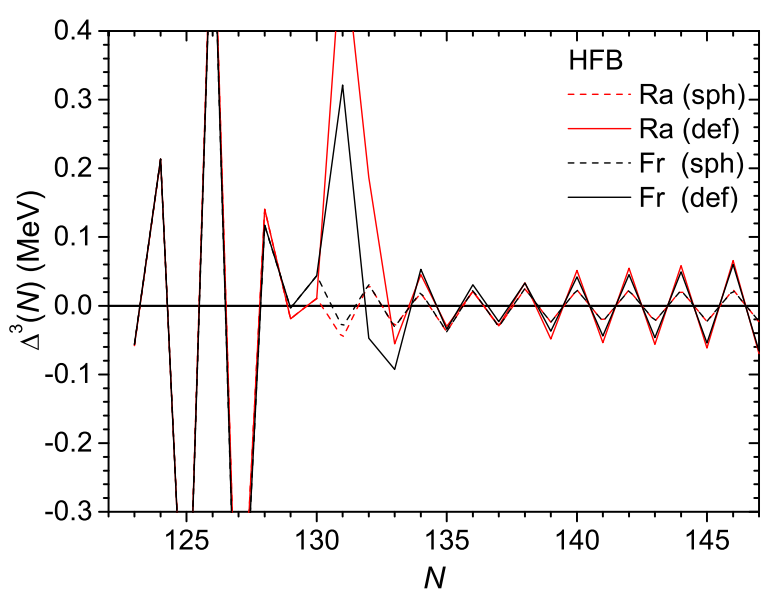

(b)

FIG. 6. (Color online) Three-point estimator of the pairing gap $\Delta^{3}(N)$ calculated for the francium and radium isotopes in the absence of pairing correlations (HF approach, left panel) and with pairing correlations but no quasiparticle blocking (HFB approach, right panel).

of $[6,8]$ suggest it may contain additional information about the effect of nuclear deformation on the single-particle structure of light nuclei. The convergence of the calculations for odd- $N$ nuclides is on the order of $50-100 \mathrm{keV}$, sufficient for drawing conclusions on the general trend, but insufficient for directly studying the staggering of $\Delta^{3}(N)$. To achieve this, the contribution of pairing correlations would have to be removed as in [6] or a calculation without blocking has to be performed [10].

In a second step, the effect of deformation on the three-point estimator $\Delta^{3}(N)$ is investigated. To suppress the contribution of pairing correlations to the odd-even staggering, Satula et al. [6] performed a pure deformed Hartree-Fock (HF) calculation. Nuclear deformation breaks rotational symmetry and lifts the $2 j+1$ degeneracy of the spherical levels. The resulting Nilsson orbits can only be occupied by two particles each. Thus, for every second particle, a new Nilsson orbit of different single-particle energy starts to be filled. In a pure single-particle picture, a corresponding energy gap is produced for every even- $N$ nuclide. The variation of the neutron Fermi level (or chemical potential) $\lambda$ with $N$ in the HF approximation is connected to the second derivative of the binding energy via

$$
\left(\frac{\partial^{2} E_{0}}{\partial N^{2}}\right)_{N_{0}}=\left(\frac{\partial \lambda}{\partial N}\right)_{N_{0}} \approx \frac{1}{2}\left[1+(-1)^{N_{0}}\right]\left(e_{N_{0}+1}-e_{N_{0}}\right)
$$

where $e_{N_{0}}$ represents the energy of the last-occupied Nilsson orbit. To study the effects of deformation on the three-point estimator $\Delta^{3}(N)$ in the HF approach, Eq. (9) is substituted into Eq. (8) yielding for $\Delta^{3}(N)$

$$
\Delta^{3}\left(N_{0}\right)=\frac{1}{4}\left[1+(-1)^{N_{0}}\right]\left(e_{N_{0}+1}-e_{N_{0}}\right) .
$$

The results of Satula et al. predict a $\Delta^{3}(N)$ close to 0 for odd- $N$ and large for even- $N$. Following their approach [6], Fig. 6(a) shows the comparison of the calculated $\Delta^{3}(N)$ to the expected value given by the right-hand side of Eq. (10), which represents the spacing in the deformed single-particle energy spectrum. For the even- $N$ radium isotopes, this would be half the spacing between the Fermi level (green line of Fig. 4) and the next orbit in the Nilsson spectrum. If $\Delta^{3}(N)$ was solely a measure of the empirical pairing gap, it should be zero everywhere in a HF calculation, which is true only for the odd- $N$ values. The even- $N$ values, however, differ from zero and their magnitude is in agreement with the interpretation of Satula et al., represented by Eq. (10). The general odd-even staggering effect is lower than the one observed in the lightmass region (cf. Fig. 1 of [6]) as expected due to the higher density of levels in heavy nuclei.

Taking into account Figs. 3 and 4, the association of the single-particle picture to the behavior of finite-difference formulas in the HF approximation becomes apparent. The large gap in the single-particle energy spectrum observed in Fig. 4 between $N=132$ and $N=133$, for example, determines a large value of $\Delta^{3}(N)$ at $N=132$ and a drop in $S_{2 N}$ between $N=132$ and $N=134$. In the region between $N=133$ and $N=138$, the occupation of energetically higher-lying Nilsson orbits is compensated by their lowering due to the increase of quadrupole deformation; see the upper abscissa of Fig. 4. This translates into a decrease of the staggering of $\Delta^{3}(N)$ and a flattening of the trend of $S_{2 N}$. Finally, the gaps above $N=140$ and $N=144$ in the Nilsson diagram translate into a sudden increases in the slope of $S_{2 N}$ and correspond to the higher peaks of $\Delta_{3}(N)$. In the francium isotopic chain, the emerging bump in the experimental $S_{2 N}$ values between $N=140$ and $N=146$ would be interpreted in the deformed single-particle picture as the proximity of several consecutive Nilsson orbits around the Fermi level.

However tempting this approach for investigating the single-particle structure of exotic nuclei might be, a few important differences have to be taken into account when passing from the pure HF picture to the fully (pairing plus quadrupole) correlated system, as pointed out in [1,10]. First, going from the no-pairing limit to the full treatment of pairing correlations mixes the pure single-particle information and smoothes the trends visible in nuclear binding energies. 

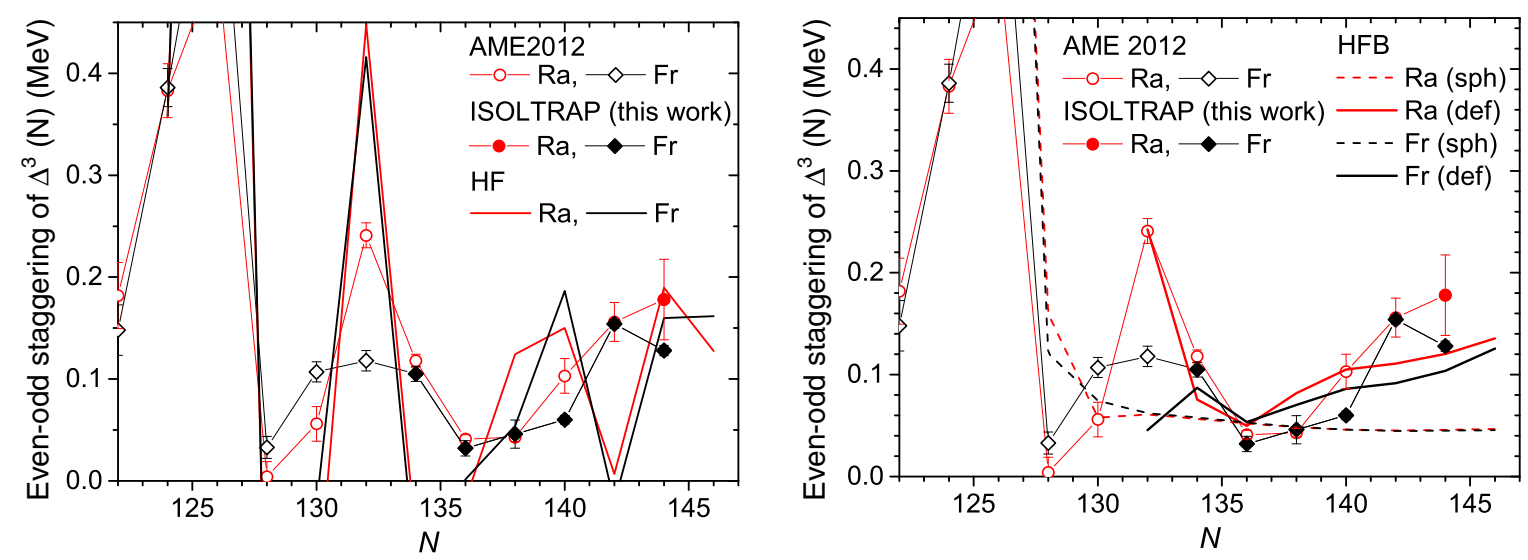

FIG. 7. (Color online) Drop between even and odd values of the empirical pairing gap $\tilde{\Delta}^{3}\left(N_{\text {even }}\right)$ for experimental values and for the calculated values of Fig. 6. The left panel shows calculations in the HF approximation, while the right panel shows the same comparison for HFB calculations without quasiparticle blocking.

Second, pairing correlations favor sphericity, thus changing the equilibrium deformation of mid-shell nuclei with respect to the pure HF result. The comprehensive analysis of [10] applied to the cerium isotopic chain casts doubt on the possibility of connecting the OES to the underlying single-particle picture (with few exceptions).

To investigate the changes, which result when passing from the HF to the pairing-correlated picture, $\Delta^{3}(N)$ is calculated in the HFB approximation following the approach of [10] without quasiparticle blocking. In this case, the value of $\Delta^{3}(N)$ does not contain the contribution of the blocking gap, but only that of the second term on the right-hand side of Eq. (8), whose dominant part is the second derivative of the ground-state energy. It thus allows to study "mean-field" contributions to $\Delta^{3}(N)$ without requiring the suppression of pairing correlations. The results are shown in Fig. 6(b) for the radium and francium isotopes. Calculating $\Delta^{3}(N)$ for the spherical and for the deformed solutions allows to quantify the effect of deformation. One notices that $\Delta^{3}(N)$ has a symmetric staggering around the value of the blocking gap (in this case zero) even for the spherical solution without having enforced the independent single-particle picture or breaking rotational symmetry. Allowing for deformation produces the kink at $N=132$, corresponding to the onset of quadrupole deformation (see also Fig. 3), and increases the staggering of $\Delta^{3}(N)$ from $N=140$ to $N=146$.

To compare theoretical results to experimental values of Fig. 5, the magnitude of the drop after each even- $N$ value of the empirical pairing gap $\tilde{\Delta}^{3}\left(N_{\text {even }}\right)$

$$
\tilde{\Delta}^{3}\left(N_{\text {even }}\right)=\Delta^{3}\left(N_{\text {even }}\right)-\Delta^{3}\left(N_{\text {even }}+1\right)
$$

is calculated and shown in Fig. 7(a) for the HF and in Fig. 7(b) for the HFB calculations. The deformed HFB results are not shown for $N \leqslant 132$ because the suddenness of the onset of deformation produces unphysical results. Both approaches predict the enhancement of the odd-even staggering of $\Delta^{3}(N)$ towards $N=146$ as an effect of quadrupole deformation. The better agreement of the HFB picture supports the importance of pairing correlations in correctly describing the trend of nuclear binding energies and underlines the difficulty of establishing an unambiguous link to an underlying single-particle picture.

Still, traces of the single-particle level density might directly reflect on the mass surface. The inclusion of dynamic quadrupole correlations beyond the static mean-field picture, with an exact restoration of particle-number and rotational symmetries, is required to clarify the impact of pairing and quadrupole correlations on the OES of binding energies and of $\Delta^{3}(N)$. Such calculations have already been performed, but are currently limited to even-even nuclei [54].

\section{CONCLUSIONS AND OUTLOOK}

The masses of neutron-rich ${ }^{222,224,226-233} \mathrm{Fr}$ and ${ }^{233,234} \mathrm{Ra}$ have been determined to a relative precision below $10^{-7}$ with the ISOLTRAP Penning-trap mass spectrometer and the ISOLDE facility at CERN. In the case of ${ }^{233} \mathrm{Fr}$, mass and half-life were determined for the first time. The mass measurements presented here extend our knowledge of the francium and radium isotopic chains to $N=146$.

We have performed $\mathrm{HF}(B)$ calculations with the SLy4 interaction $[48,49]$ using the HFODD code $[47,50]$ to understand the impact of quadrupole and octupole correlations on the twoneutron separation energies and the three-point pairing-gap estimator $\Delta^{3}(N)$ in the case of heavy nuclides. The Nilsson level scheme between $N=128$ and $N=148$ extracted from the HF calculations exhibits a near degeneracy of orbits which favors an enhancement of octupole collectivity. Together with HFB results from literature, which include octupole correlations [55], this leads to the conclusion that the full magnitude of the kink in $S_{2 N}$ at $N=132$ is due to the combination of quadrupole and octupole correlations.

Following previous approaches $[1,6,8-10]$, the heavymass region was investigated to illustrate the contribution of pairing correlations and nuclear deformation to the OES of binding energies as well as to the staggering of $\Delta^{3}(N)$. HFB calculations with and without quasiparticle blocking have shown that nuclear deformation influences the OES and $\Delta^{3}(N)$. Including quadrupole deformation leads to a reasonable description of $\Delta^{3}(N)$ towards $N=146$. In the 
HF no-pairing limit, the internal link between $\Delta^{3}(N)$ and the single-particle picture [6] has been verified, one example being the large gap above the Fermi level observed in the HF calculation around $N=132$, which corresponds to a local enhancement of the staggering of $\Delta^{3}(N)$ also present in experiment. The comparison of calculations to data can also be polluted by imperfect sequences of single-particle orbits which might affect details of the pairing strength. Thus, the link might be restricted to a limited number of cases taking into account the precision of experimental data.

The new data revealed an enhanced staggering of $\Delta^{3}(N)$ towards $N=146$, which could be reproduced by both $\mathrm{HF}$ and HFB calculations, as an effect of quadrupole deformation. Quantitatively, the HFB picture is in better agreement, corroborating the importance of pairing correlations. It would be interesting to extend the existing charge radii OES measurements as far as the mass measurements to challenge directly the importance of quadrupole deformation in those nuclei.

\section{ACKNOWLEDGMENTS}

We thank G. Bertsch, S. Goriely, and L. Robledo for communications. We kindly thank J. Dobaczewski for discussions and comments on the manuscript. This work was supported by the BMBF under Contracts No. 05P12HGCI1, No. 05P12HGFNE, and No. 05P09ODCIA, the EU through ENSAR (Grant No. 262010), the French IN2P3, the ISOLDE Collaboration, and the Max-Planck Society. S.K. acknowledges support from the Robert-Bosch Foundation.
[1] M. Bender, K. Rutz, P.-G. Reinhard, and J. A. Maruhn, Eur. Phys. J. A 8, 59 (2000).

[2] J. Bonn, G. Huber, H. J. Kluge, L. Kugler, and O. E. W, Phys. Lett. B 38, 308 (1972).

[3] A. Coc, C. Thibault, F. Touchard, H. T. Duong, P. Juncar, S. Liberman, J. Pinard, J. Lermé, J. L. Vialle, S. Büttgenbach, and A. C. Mueller, Phys. Lett. B 163, 66 (1985).

[4] S. A. Ahmand, W. Klempt, R. Neugart, E. W. Otten, P. G. Reinhard, G. Ulm, and K. Wendt, Nucl. Phys. A 483, 244 (1988).

[5] J. Bardeen, L. N. Cooper, and J. R. Schriefer, Phys. Rev. 108, 1175 (1957).

[6] W. Satula, J. Dobaczewski, and W. Nazarewicz, Phys. Rev. Lett. 81, 3599 (1998).

[7] K. Rutz, M. Bender, P.-G. Reinhard, and J. A. Maruhn, Phys. Lett. B 468, 1 (1999).

[8] J. Dobaczewski, P. Magierski, W. Nazarewicz, W. Satuła, and Z. Szymański, Phys. Rev. C 63, 024308 (2001).

[9] T. Duguet, P. Bonche, P.-H. Heenen, and J. Meyer, Phys. Rev. C 65, 014310 (2001).

[10] T. Duguet, P. Bonche, P.-H. Heenen, and J. Meyer, Phys. Rev. C 65, 014311 (2001).

[11] L. M. Robledo, R. Bernard, and G. F. Bertsch, Phys. Rev. C 86, 064313 (2012).

[12] J. Hakala, J. Dobaczewski, D. Gorelov, T. Eronen, A. Jokinen, A. Kankainen, V. S. Kolhinen, M. Kortelainen, I. D. Moore, H. Penttilä, S. Rinta-Antila, J. Rissanen, A. Saastamoinen, V. Sonnenschein, and J. Äystö, Phys. Rev. Lett. 109, 032501 (2012).

[13] L. Coraggio, A. Covello, A. Gargano, and N. Itaco, Phys. Rev. C 88, 041304 (2013).

[14] Y. A. Litvinov, T. J. Bürvenich, H. Geissel, Y. N. Novikov, Z. Patyk, C. Scheidenberger, F. Attallah, G. Audi, K. Beckert, F. Bosch, M. Falch, B. Franzke, M. Hausmann, T. Kerscher, O. Klepper, H.-J. Kluge, C. Kozhuharov, K. E. G. Löbner, D. G. Madland, J. A. Maruhn, G. Münzenberg, F. Nolden, T. Radon, M. Steck, S. Typel, and H. Wollnik, Phys. Rev. Lett. 95, 042501 (2005).

[15] C. A. Bertulani, H. F. Lü, and H. Sagawa, Phys. Rev. C 80, 027303 (2009).

[16] H. Alvarez-Pol, J. Benlliure, E. Casarejos, L. Audouin, D. Cortina-Gil, T. Enqvist, B. Fernandez-Dominguez, A. R.
Junghans, B. Jurado, P. Napolitani, J. Pereira, F. Rejmund, K.-H. Schmidt, and O. Yordanov, Phys. Rev. C 82, 041602(R) (2010).

[17] P. A. Butler and W. Nazarewicz, Rev. Mod. Phys. 68, 349 (1996).

[18] W. Heisenberg, Z. Phys. 77, 1 (1932).

[19] W. A. Heer, Rev. Mod. Phys. 65, 611 (1993).

[20] C. T. Black, D. C. Ralph, and M. Tinkham, Phys. Rev. Lett. 76, 688 (1996)

[21] D. C. Ralph, C. T. Black, and M. Tinkham, Phys. Rev. Lett. 78, 4087 (1997).

[22] M. Mukherjee, D. Beck, K. Blaum, G. Bollen, J. Dilling, S. George, F. Herfurth, A. Herlert, A. Kellerbauer, H.-J. Kluge, S. Schwarz, L. Schweikhard, and C. Yazidjian, Eur. Phys. J. A 35, 1 (2008).

[23] E. Kugler, Hyperfine Interact. 129, 23 (2000).

[24] S. Kreim, D. Atanasov, D. Beck, K. Blaum, C. Böhm, C. Borgmann, M. Breitenfeldt, T. E. Cocolios, D. Fink, S. George, A. Herlert, A. Kellerbauer, U. Köster, M. Kowalska, D. Lunney, V. Manea, E. Minaya Ramirez, S. Naimi, D. Neidherr, T. Nicol, R. E. Roessel, M. Rosenbusch, L. Schweikhard, J. Stanja, F. Wienholtz, R. N. Wolf, and K. Zuber, Nucl. Instrum. Methods B 317, 492 (2013).

[25] F. Herfurth, J. Dilling, A. Kellerbauer, G. Bollen, S. Henry, H.-J. Kluge, E. Lamour, D. Lunney, R. B. Moore, C. Scheidenberger, S. Schwarz, G. Sikler, and J. Szerypo, Nucl. Instrum. Methods A 469, 254 (2001).

[26] R. N. Wolf, D. Beck, K. Blaum, C. Böhm, C. Borgmann, M. Breitenfeldt, F. Herfurth, A. Herlert, M. Kowalska, S. Kreim, D. Lunney, S. Naimi, D. Neidherr, M. Rosenbusch, L. Schweikhard, J. Stanja, F. Wienholtz, and K. Zuber, Nucl. Instrum. Methods A 686, 82 (2012).

[27] N. Bradbury and R. Nielsen, Phys. Rev. 49, 388 (1936).

[28] R. Wolf, F. Wienholtz, D. Atanasov, D. Beck, K. Blaum, C. Borgmann, F. Herfurth, M. Kowalska, S. Kreim, Y. A. Litvinov, D. Lunney, V. Manea, D. Neidherr, M. Rosenbusch, L. Schweikhard, J. Stanja, and K. Zuber, Int. J. Mass Spectrom. 349-350, 123 (2013).

[29] G. Savard, S. Becker, G. Gollen, H.-J. Kluge, R. B. Boore, T. Otto, L. Schweikhard, H. Stolzenberg, and U. Wiess, Phys. Lett. A 158, 247 (1991). 
[30] S. Becker, G. Bollen, F. Kern, H.-J. Kluge, R. Moore, G. Savard, L. Schweikhard, and H. Stolzenberg, Int. J. Mass Spectrom. Ion Process. 99, 53 (1990).

[31] G. Gräff, H. Kalinowsky, and J. Traut, Z. Phys. A 297, 35 (1980).

[32] G. Bollen, R. B. Moore, G. Savard, and H. Stolzenberg, J. Appl. Phys. 68, 4355 (1990).

[33] M. König, G. Bollen, H.-J. Kluge, T. Otto, and J. Szerypo, Int. J. Mass. Spectrom. Ion Process. 142, 95 (1995).

[34] A. Kellerbauer, K. Blaum, G. Bollen, F. Herfurth, H.-J. Kluge, M. Kuckein, E. Sauvan, C. Scheidenberger, and L. Schweikhard, Eur. Phys. J. D 22, 53 (2003).

[35] G. Audi, F. G. Kondev, M. Wang, B. Pfeiffer, X. Sun, J. Blachot, and M. MacCormick, Chin. Phys. C 36, 1157 (2012).

[36] G. Audi, A. H. Wapstra, and C. Thibault, Nucl. Phys. A 729, 337 (2003).

[37] L. Chen, W. R. Plass, H. Geissel, R. Knöbel, C. Kozhuarov, Y. A. Litvinov, Z. Patyk, C. Scheidenberger, K. Siegien-Iwaniuk, B. Sun, H. Weick, K. Beckert, P. Beller, F. Bosch, D. Boutin, L. Caceres, J. J. Carroll, D. M. Cullen, I. J. Cullen, B. Franzke, J. Gerl, M. Gorska, G. A. Jones, A. Kishada, J. Kurcewicz, S. A. Litvinov, Z. Liu, S. Mandal, F. Montes, G. Münzenberg, F. Nolden, T. Ohtsubo, Z. Podolyak, R. Propri, S. Rigby, N. Saito, T. Saito, M. Shindo, M. Steck, P. M. Walker, S. Williams, M. Winkler, H.-J. Wollersheim, and T. Yamaguchi, Nucl. Phys. A 882, 71 (2012).

[38] F. Herfurth, G. Audi, D. Beck, K. Blaum, G. Bollen, P. Delahaye, S. George, C. Guenaut, A. Herlert, A. Kellerbauer, H.-J. Kluge, D. Lunney, M. Mukherjee, S. Rahaman, S. Schwarz, L. Schweikhard, C. Weber, and C. Yazidijan, Eur. Phys. J. A 25, 17 (2005).

[39] M. Wang, G. Audi, A. H. Wapstra, F. G. Kondev, M. MacCormick, X. Xu, and B. Pfeiffer, Chin. Phys. C 36, 1603 (2012).

[40] G. Bollen, H.-J. Kluge, T. Otto, G. Savard, L. Schweikhard, H. Stolzenberg, G. Audi, R. B. Moore, and G. Rouleau, J. Mod. Opt. 39, 257 (1992).

[41] C. Weber, G. Audi, D. Beck, K. Blaum, G. Bollen, F. Herfurth, A. Kellerbauer, H.-J. Kluge, D. Lunney, and L. Schweikhard, Nucl. Phys. A 803, 1 (2008).

[42] D. Neidherr, G. Audi, D. Beck, K. Blaum, Ch. Böhm, M. Breitenfeldt, R. B. Cakirli, R. F. Casten, S. George, F. Herfurth, A. Herlert, A. Kellerbauer, M. Kowalska, D. Lunney, E. MinayaRamirez, S. Naimi, E. Noah, L. Penescu, M. Rosenbusch, S. Schwarz, L. Schweikhard, and T. Stora, Phys. Rev. Lett. 102, 112501 (2009).
[43] H. Klapdor, J. Metzinger, and T. Oda, At. Data Nucl. Data Tables 31, 81 (1984).

[44] A. Staudt, E. Bender, K. Muto, and H. Klapdor-Kleingrothaus, At. Data Nucl. Data Tables 44, 79 (1990).

[45] P. Moller, J. R. Nix, and K.-L. Kratz, At. Data Nucl. Data Tables 66, 131 (1997).

[46] M. Bender, P.-H. Heenen, and P.-G. Reinhard, Rev. Mod. Phys. 75, 121 (2003).

[47] N. Schunck, J. Dobaczewski, J. McDonnel, W. Satula, J. Sheikh, A. Staszczak, M. Stoitsov, and P. Toivanen, Comput. Phys. Commun. 183, 166 (2012).

[48] E. Chabanat, P. Bonche, P. Haensel, J. Meyer, and R. Schaeffer, Nucl. Phys. A 635, 231 (1998).

[49] E. Chabanat, P. Bonche, P. Haensel, J. Meyer, and R. Schaeffer, Nucl. Phys. A 643, 441 (1998).

[50] J. Dobaczewski, W. Satuła, B. Carlsson, J. Engel, P. Olbratowski, P. Powałowski, M. Sadziak, J. Sarich, N. Schunck, A. Staszczak, M. Stoitsov, M. Zalewski, and H. Zduńczuk, Comput. Physi. Commun. 180, 2361 (2009).

[51] V. Manea, D. Atanasov, D. Beck, K. Blaum, C. Borgmann, R. B. Cakirli, T. Eronen, S. George, F. Herfurth, A. Herlert, M. Kowalska, S. Kreim, Y. A. Litvinov, D. Lunney, D. Neidherr, M. Rosenbusch, L. Schweikhard, F. Wienholtz, R. N. Wolf, and K. Zuber, Phys. Rev. C 88, 054322 (2013).

[52] L. Gaffney, P. A. Butler, M. Scheck, A. B. Hayes, F. Wenander, M. Albers, B. Bastin, B. C, A. Blazhev, S. Bönig, N. Bree, J. Cederkäll, T. Chupp, D. Cline, T. E. Cocolios, T. Davinson, H. De Witte, J. Diriken, T. Grahn, A. Herzan, M. Huyse, D. G. Jenkins, D. T. Joss, N. Kesteloot, J. Konki, M. Kowalczyk, T. Kröll, E. Kwan, R. Lutter, K. Moschner, P. Napiorkowski, J. Pakarinen, M. Pfeiffer, D. Radeck, P. Reiter, K. Reynders, S. V. Rigby, L. M. Robledo, M. Rudigier, S. Sambi, M. Seidlitz, B. Siebeck, T. Stora, P. Thoele, P. Van Duppen, M. J. Vermeulen, M. von Schmid, D. Voulot, N. Warr, K. Wimmer, K. Wrzosek-Lipska, C. Y. Wu, and M. Zielinska, Nature (London) 497, 199 (2013).

[53] S. Hilaire and M. Girod, Eur. Phys. J. A 33, 237 (2007).

[54] M. Bender, G. F. Bertsch, and P.-H. Heenen, Phys. Rev. C 73, 034322 (2006).

[55] L. M. Robledo and G. F. Bertsch, Phys. Rev. C 84, 054302 (2011).

[56] M. Zalewski, J. Dobaczewski, W. Satuła, and T. R. Werner, Phys. Rev. C 77, 024316 (2008).

[57] D. G. Madland and J. R. Nix, Nucl. Phys. A 476, 1 (1988). 